This item was submitted to Loughborough's Research Repository by the author.

Items in Figshare are protected by copyright, with all rights reserved, unless otherwise indicated.

\title{
A study of different loss of life based calculations on batteries operating in enhanced frequency response
}

PLEASE CITE THE PUBLISHED VERSION

https://doi.org/10.1109/UPEC.2018.8542094

\section{PUBLISHER}

(C) European Union. Published by IEEE

\section{VERSION}

AM (Accepted Manuscript)

\section{PUBLISHER STATEMENT}

Personal use of this material is permitted. Permission from IEEE must be obtained for all other uses, in any current or future media, including reprinting/republishing this material for advertising or promotional purposes, creating new collective works, for resale or redistribution to servers or lists, or reuse of any copyrighted component of this work in other works.

\section{LICENCE}

\section{All Rights Reserved}

\section{REPOSITORY RECORD}

Nute, Maria L.G., Dani Strickland, and Mina Abedi-Varnosfaderani. 2019. "A Study of Different Loss of Life Based Calculations on Batteries Operating in Enhanced Frequency Response". figshare. https://hdl.handle.net/2134/38105. 


\section{A study of different loss of life based calculations on batteries operating in enhanced frequency response}

\author{
Heather Nute \\ Department of Physics \\ Loughborough University \\ Loughborough, UK \\ heatheranute@gmail.com
}

\author{
Dani Strickland \\ Wolfson School \\ Loughborough University \\ Loughborough, UK \\ d.strickland@lboro.ac.uk
}

\author{
Mina Abedi-Varnosfaderani \\ Wolfson School \\ Loughborough University \\ Loughborough, UK \\ m.abedi-varnosfaderani@lboro.ac.uk
}

\begin{abstract}
National Grid are investigating battery energy storage as a solution to help with energy balancing for frequency control. They are currently looking at Enhanced Frequency Response (EFR) as a solution, but, are unlikely to adapt this directly as they investigate new, more open, market strategies. Battery Energy Storage is still an expensive asset and the opportunity to use a hybrid scheme (with new and second life batteries) offers a potential lower cost solution. Determining the life cycle of a battery energy storage system is complex, as this is a combination of several processes including cyclic and calendric aging. Determining life cycles of a hybrid system is even more complex. This paper discusses different ways of determining life span published in literature and uses a model based case study approach using National Grid published data on frequency and EFR envelopes to investigate the different range of theoretical loss of life within different battery system.
\end{abstract}

Keywords - Energy Storage, EFR, FFR, Degradation, Batteries

\section{INTRODUCTION}

National Grid are required to keep stability in the UK grid system. To this end they have to balance generation with demand. As more renewable generation comes on-line they are attempting to solve issues with reduced grid stability through the use of battery energy storage offering grid frequency support services. Battery energy storage is expensive, thus it is difficult to establish a business case. One of the key inputs into sizing and quantifying the system is the likely impact of degradation. There is discussion on using hybrid battery systems with a combination of new and second life (extransportation batteries) to meet the service at a lower cost than with just a new battery.

This work uses frequency data published by National Grid in conjunction with their planned service envelopes to establish what impact this type of response is likely to have on the degradation of different types of batteries, as this significantly impacts the business case for purchasing batteries. To help control frequency National Grid use four different main methods:

- Mandatory Frequency Response

- $\quad$ Firm Frequency Response

- Demand Management Frequency Control

- $\quad$ Firm Frequency Response Bridging [1]
Firm frequency response services in the UK are known as Primary response, Secondary response and High response; Primary response is the automatic increase in active power output of a generator or the automatic decrease in demand in response to a fall in system frequency which must be fully available within 10 seconds of the frequency fall and sustainable for a further 20 seconds. Primary response has an approximate 2 second delay in responding to an event. Secondary response can then be used to restore the frequency over a longer time period, while High frequency response is connected with backing off generation or increasing demand in the event that the frequency is too high. Although these control mechanisms have been used successfully for many years, the system is undergoing transformation with the implementation of the low carbon agenda, which is increasing the amount of low inertia renewable generation within the system [2]. To help with this issue National Grid are trialling a new service Enhanced Frequency Response (EFR) to assess its suitability [3]. However, it appears that National Grid and its frequency service providers are struggling with the complexity of the addition of potentially different market streams such as EFR and in particular how they relate to the energy storage market. A movement to simplify the structure is therefore underway [4]. To this end, it is not yet clear what the future frequency response requirements will take. This uncertainty complicates the requirement to determine the business case for adding Energy Storage to the Grid, as the operational requirements impact their life span and subsequent replacement/maintenance costs. However, as Energy Storage is fast acting and has the capability to both charge and discharge, it is likely that some form of EFR type response will be included in the new market structure to benefit from the advantages of battery energy storage systems. The battery will therefore not be charging and discharging under a fixed operating regime - but will be going through a micro-cycle type operation as it charges and discharges in response to the frequency. Previous literature has looked into micro-cycles of vehicles and renewable generation but hasn't explicitly considered EFR response applications.

This paper therefore uses the EFR envelope as a basis for calculating the theoretical impact on life span of different battery types, under different means of calculating degradation through micro-cycles. This gives an idea as to the variability in life cycle determination under a micro-cycle based operating 
program. Section II examines the application, Section III considers micro-cycling impacts from this, while Section IV considers a case study approach to show the range of loss of life which may be calculated due to micro-cycles.

\section{ENHANCED FREQUENCY RESPONSE}

\section{A. EFR Background}

The Enhanced Frequency Control Capability project is a research based, Low Carbon Network Funded (LCNF) project being undertaken by National Grid [3]. One aim of the project is to help investigate enhanced frequency control in view of falling system inertia as an alternative to increasing the current availability of current response methods (which would be costly). National Grid published the first technical requirements for EFR battery storage in 2015, with a follow up in 2016. The specifications are in graphical form, providing the amount of response required for a specific frequency value (see Error! Reference source not found. ).

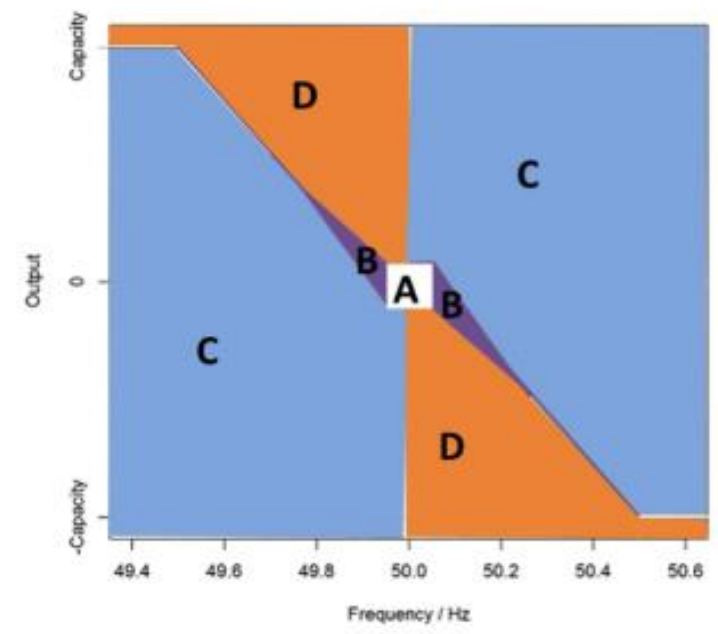

Fig 1. Enhanced frequency response envelope.

On the $\mathrm{x}$-axis is the frequency. The graph provides a specification for the deviation from the nominal $50 \mathrm{~Hz}$ value. On the y-axis is the per unit capacity of the battery storage. There are two specifications (wide and narrow) detailing the response of the energy storage system -in this work only the wide band will be used as the metering for the narrow band is not commonly available. Battery State of charge (SOC) management is allowed within the dead band area and the areas either side of this with the branching lines. The management of the SOC has been investigated in several bodies of work [5,6] and introduces an additional complexity when undertaking analysis into micro-cycles. In this work a simplified version of SOC management has been chosen so as not to over complicate the micro-cycle analysis. However future work could look at the impact of SOC management on life cycle analysis. The SOC management strategy and response to the published frequency data is used to set the battery behaviour from which the micro-cycle degradation response can be calculated.
In addition to this. National Grid also published the frequency data for a full year (2014) to help providers determine an operating strategy as shown in Fig 2 .

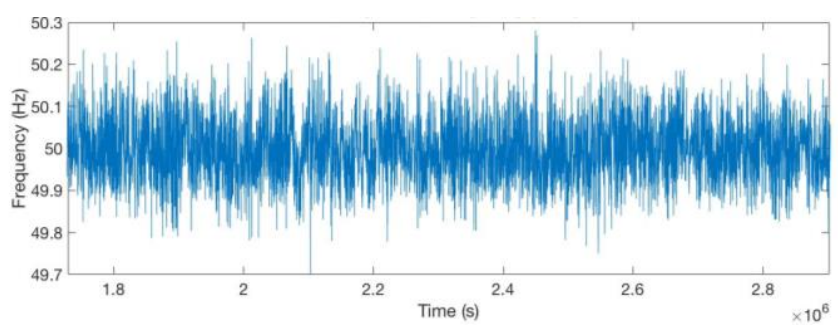

Fig 2. A sample of National Grid published frequency data [5]

\section{B. SOC management strategy}

The SOC management strategy was designed to minimise the downtime of the battery (to maximise availability). The initial starting point in this was to determine which SOC was best to set the battery to within the dead band area, such that the battery required the minimum number of recharging events while following the response curve, with no further intervention.MATLAB (R2017a) was used to import the reported grid frequency data for 2014 from the National Grid website in CSV format in monthly files. A starting point for battery SOC was chosen as shown in Fig 3 (the point where the battery sits in location A in Error! Reference source not found. waiting for a frequency event) such that if in the act of providing a grid service the battery goes above or below its operating limits it can reset back to this value. During this process the battery is assumed to be unavailable for service. In this way the total time that the battery is out of service can be calculated for each starting SOC. No complex battery management process in location $\mathrm{B}$ is assumed to occur at this time. The battery follows the lower line of service for an under frequency event and the upper line for an over frequency event.This is obviously not the optimum method of battery management but provides a straightforward measure of battery charging and discharging under typical EFR operating conditions. A starting SOC of $47 \%$ was chosen as the solution which gives the maximum availability to National Grid. For each frequency the power that the battery should charge and discharge was calculated from the operating envelope and the battery SOC adjusted accordingly. That way, as the time increased, the battery charged and discharged as per frequency response as shown in Fig 4

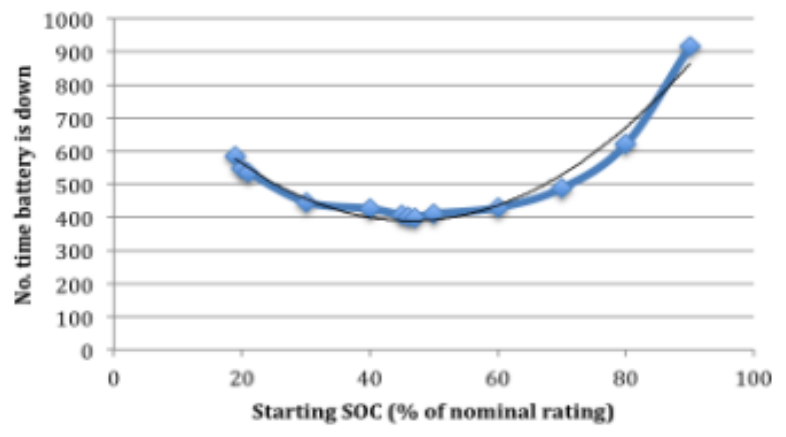

Fig 3. Graph of starting SOC vs. no of total off times for BESS. 

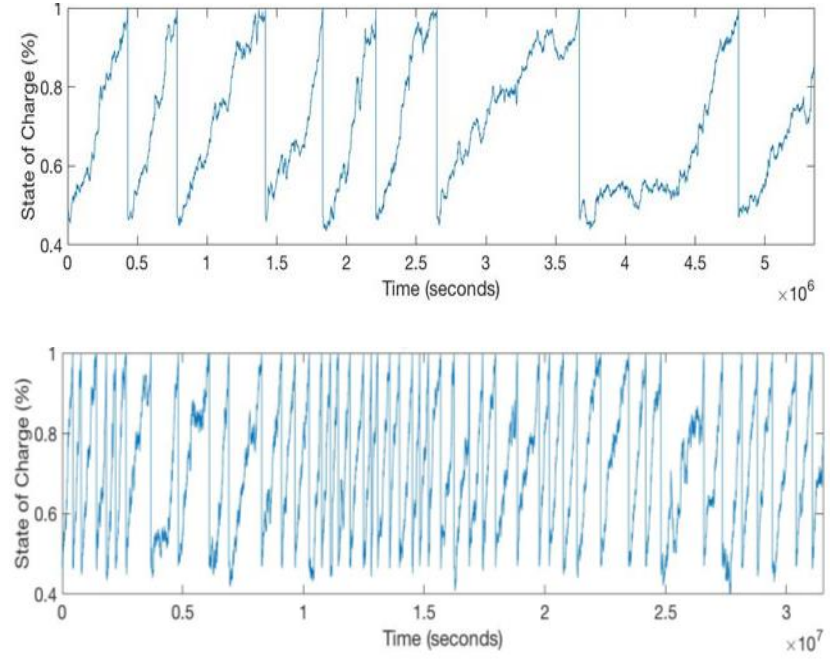

Fig 4. Graph of battery charging and discharging with time for 1 month (Jan 2014) and the full year (2014)

Fig 5 shows a close up of the graph to allow the microcycles to be seen more clearly. These are very small in comparison to the other traditionally reported micro-cycles.

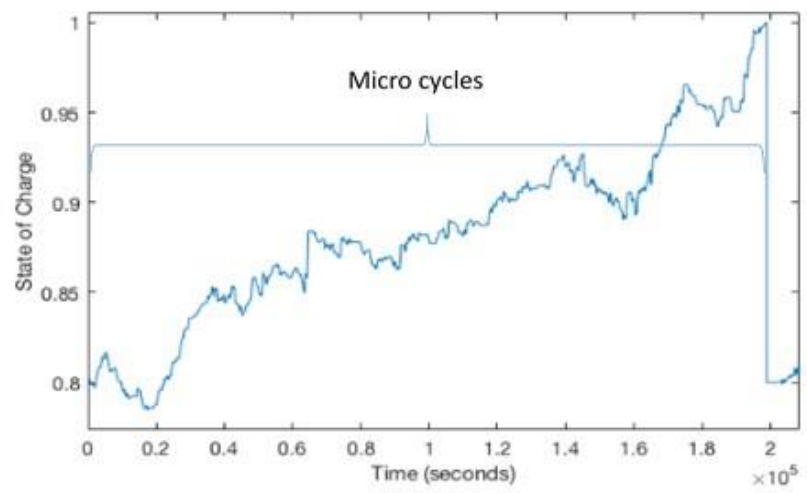

Fig 5. Zoom in on the operating graph showing the micro-cycling

\section{DEGRADATION}

There are two main types of degradation for batteries these are usually referred to as calendric and cyclic aging. Calendric aging is the effect on the lifetime of the battery as it ages over the months and years 'naturally'. Cycling aging is the deterioration of the life of the battery due to the way in which it is used or cycled. Typically, a manufacturer will provide graphs of number of cycles against depth of discharge (DOD) of a battery which gives an indication of life span. Fig 6 and Fig 7 show the Wohler curve for a lead acid and Lithium Ion battery respectively reproduced from [7] and [8] and used within this report. However, the data in Fig 5 clearly shows that the charging and discharging of the battery is not to a fixed DOD, but instead varies as a function of micro-cycles to meet the frequency requirements.

A battery micro-cycle can be defined in a number of different ways: 'micro-cycles' can be functions of polarity reversals of the battery current with periods less than minutes.
A micro-cycle can also be defined as the battery moving from a particular state of charge changing up or down by some depth of discharge and then returning to the same state of charge.

Previous analysis of micro-cycles have been defined in literature. These are primarily based around electric vehicle driving cycles as this is the main area where batteries are used which exhibit micro-cycle charge and discharge tendencies. Examples include;

- IEC 61982-2 [9] Dynamic Endurance Test - the current is set high to simulate acceleration, then low to simulate constant speed and then paused.

- ALIDISSI [10] This has a high power battery macro and micro-cycling profile. The micro-cycles are defined to represent driving phases such as cruise drive, acceleration and deceleration with regenerative braking while the macro cycles look at 22 hours microcycling enabling to reach a predetermined minimum SOC for the studied battery followed by a $2 \mathrm{~h}$ "non cycling" period.

- Other typical drive cycle type analysis [11] have included a succession of micro-cycles (i.e., discharging/charging current pulses). The micro-cycles are typically not charge-neutral in order to produce a SOC swing. Their shape is constituted by a charging pulse followed by a discharging pulse.

- Some theoretical micro-cycling profiles relating to renewable generation has also been defined [12].

These methods of defining and quantifying aging based on micro-cycle testing typically show large micro-cycles $>1 \%$ SOC and may not be directly relevant to a frequency response application as the micro-cycling is much smaller. To look more specifically at EFR produced data without the benefit of test data to refer to, an alternative approach is required. There are a number of proposed theoretical methods of estimating life cycles that are based on the Wohler curve.

The process behind these are;

- Define what is meant by a micro-cycle

- Determine when a micro-cycle occurs

- Use the Wohler curve to associate each of these microcycles with an associated loss of life. Within this work this has been done using (1)

$$
L_{i}=\frac{1}{L C_{D O D}}
$$

Where $L_{i}$ is the loss of life for the $i^{\text {th }}$ micro-cycle and $\mathrm{LC}_{\mathrm{DOD}}$ is the number of life cycles for that DOD. The total loss of life over all cycles is the sum of all the $\mathrm{L}_{\mathrm{i}}$ components.

Typically it is assumed that;

- The battery ages by the same amount in the charging and discharging process.

- The aging is independent of the previous aging of the battery and previous cycle events.

There are a number of different methods that have been developed to identify micro-cycles. [13] uses a tolerance belt approach where a wide tolerance doesn't detect small microcycles but picks up macro cycles as shown in Fig 8 . The user has to provide the width for the division of the micro-cycle and width of SOC discharges that the users wishes to filter out. 


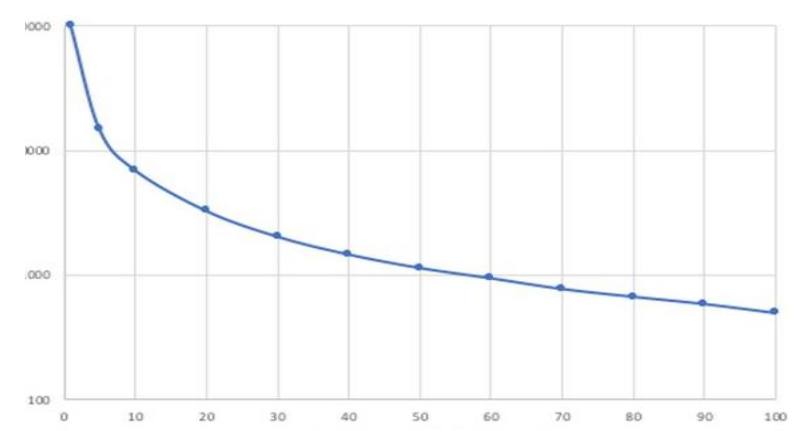

Fig 6. Graph of DOD vs life cycles of a lead acid battery [8]

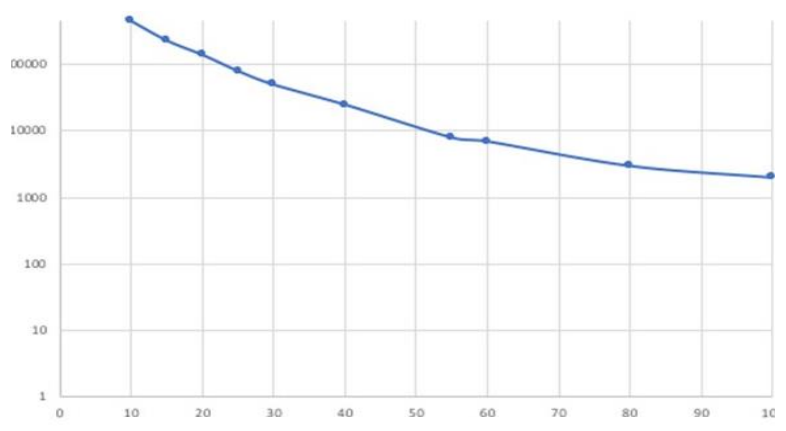

Fig 7. Graph of DOD vs life cycles of a Li-Ion battery [9]
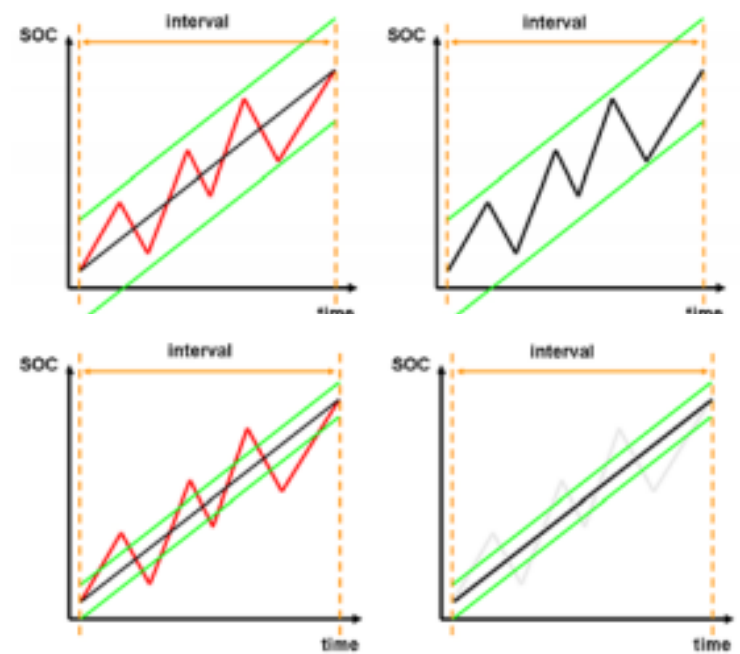

Fig 8. Tolerance belts (wide and narrow) showing no detection and detection of micro-cycles [13]

The rain flow method [14] looks at detecting the SOC full cycles and picking up the remaining cycles as residual cycles as shown in Fig 9. The loss of life is then dependent on both full and residual cycling where the residual cycling is shown by the $\delta$ values (small changes in SOC). Within the EFR operation, the change in SOC within the time scale is typically very small and much lower than $1 \%$ DOD which is typically very inaccurately defined.
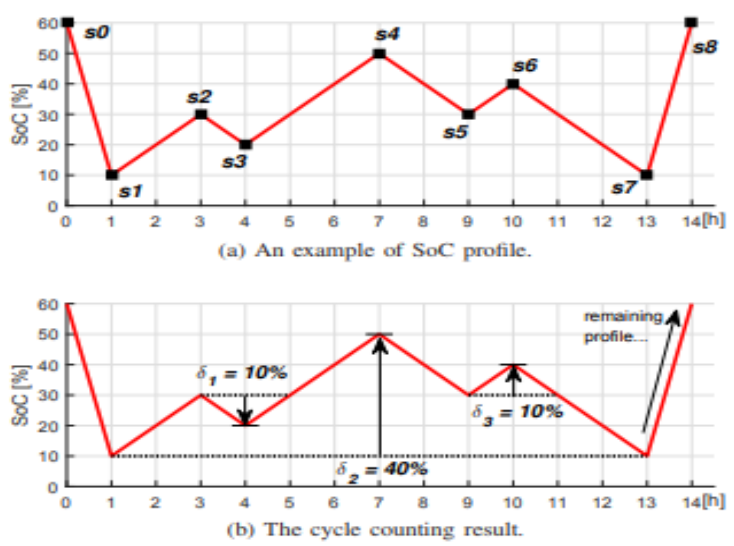

Fig 9. Rain-flow method to identify micro-cycles [14]

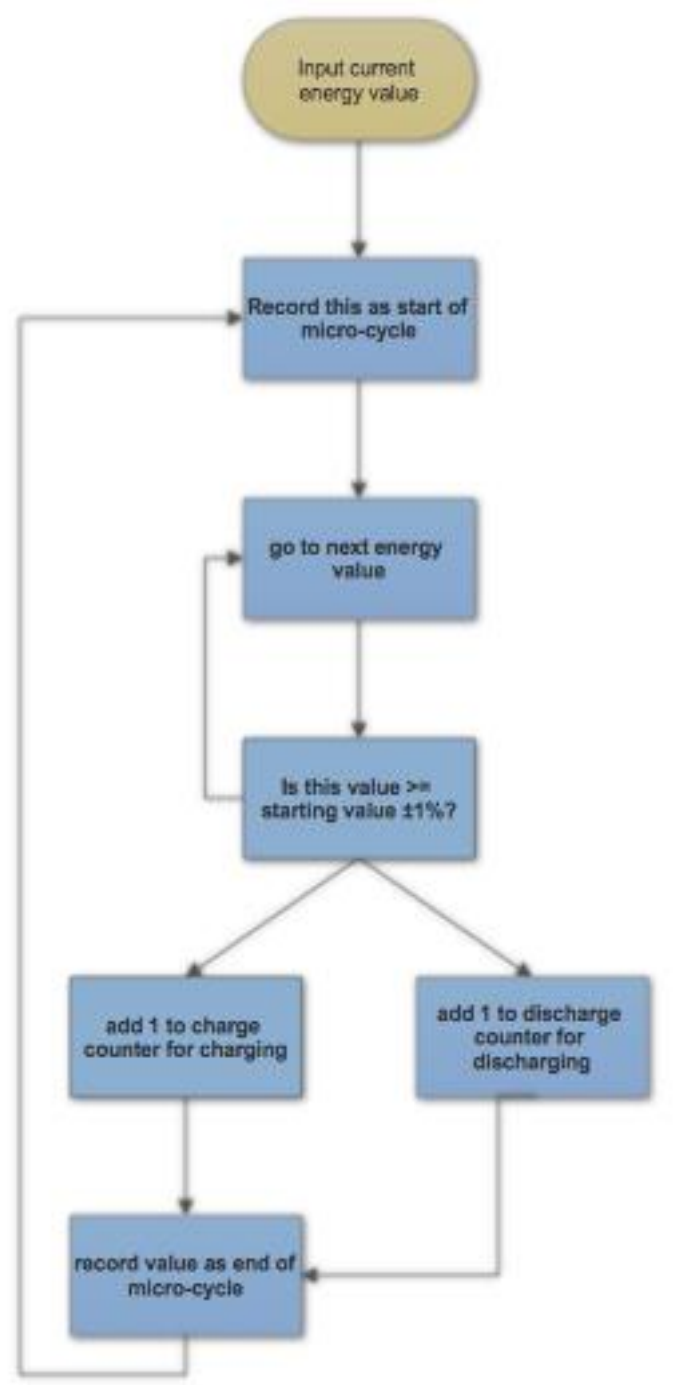

Fig 10. Determination of micro-cycles within this work.

The two methods described would thus show negligible impact on loss of life as the micro-cycle detected would be too small to realistically impact the calculation. 
In this work an alternative approach has been used. The method looks to identify when a fixed change in DOD has occurred and defines this as a micro-cycle. A micro-cycle has been therefore been defined as a single charge OR discharge of a fixed depth of discharge (set at the start by the user.) This allows omission of the difficulty of calculating what size of DoD each micro-cycle should be assigned with, although it does have the consequence that it may be slightly less accurate at determining the effect micro-cycles have on the battery life.

The flow chart in Fig 10 depicts the main processes for the counting of micro-cycles in this script. An example of this process is shown in Fig 11. The small changes in SOC over the period of 12 hours at $<1 \%$ SOC are collated by identifying only when a $1 \%$ change in SOC has been reached and are then summed. This results in a sum total of 12 micro-cycles covering $1 \%$ change in SOC as opposed to thousands of small micro-cycles in sub \%SOC variations with no means of associating a loss of life against them.
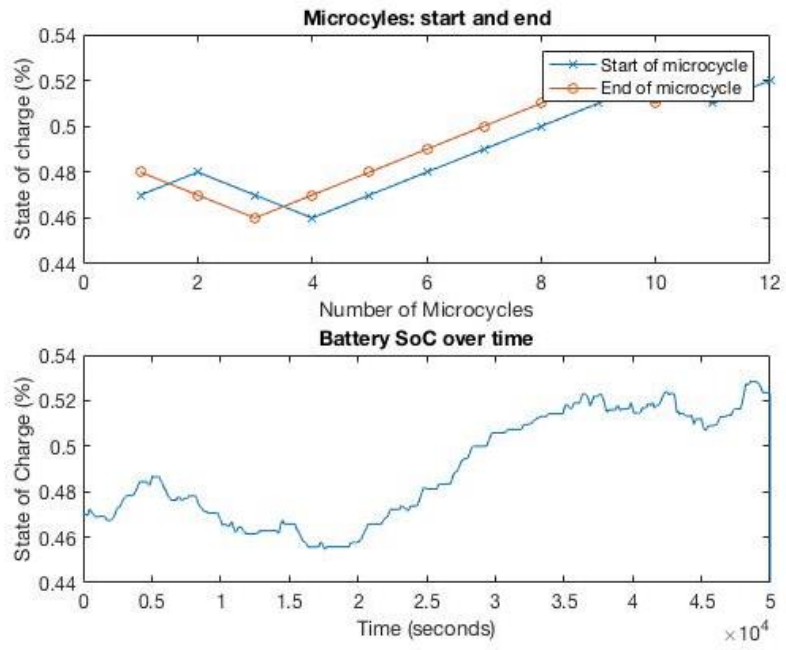

Fig 11. micro-cycles over a 12 hour period

\section{EFR CASE STUDY}

This work takes the National Grid frequency figures from Fig 2 and combines these with an SOC management strategy to determine the micro-cycles using the method in Fig 10 to allow a loss of life to be calculated through (1).

Fig 12 shows the calculation of the number of microcycles over a year long period against the SOC change of the battery system. Having identified each micro-cycle the loss of life and subsequent reduction in capacity can then be calculated from Fig 6 and Fig 7. With the capacity loss addition to the model the following results are produced based on a Lead acid battery at $1 \%$ DoD, for a lifecycle total of 100,000 cycles over a year period in Fig 12, while the equivalent data for a Li-Ion battery is shown in Fig 13.

The micro-cycling of the Lead acid battery shows a degradation of battery capacity of just over $1 \%$ over the period of a year due to the micro-cycling while the Li-Ion battery micro-cycling has negligible impact on capacity. No macro cycling events have been considered. The graphs do highlight that over the period of 2014 the battery would have spent more time charging to compensate for high frequency than discharging to compensate for low frequency. The loss of life is therefore dependent on the depth of the micro-cycle chosen as shown in Fig 14.
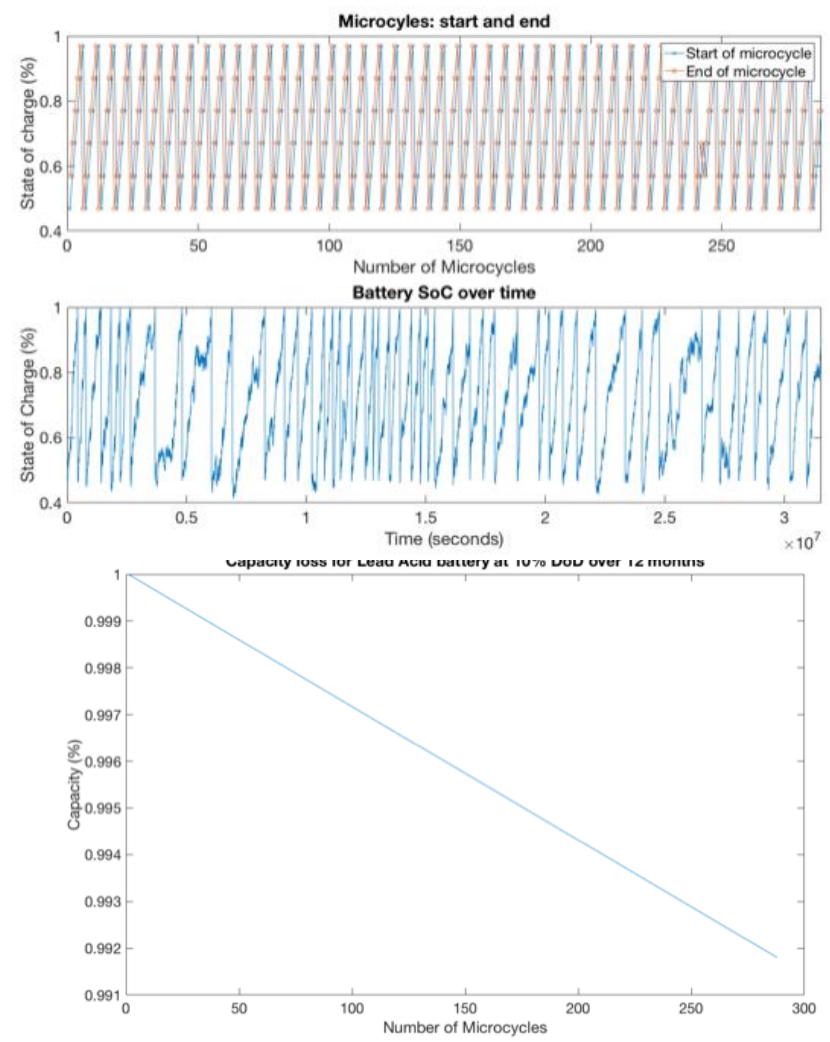

Fig 12. Lead acid battery loss of capacity with $10 \%$ DoD microcycles over a year
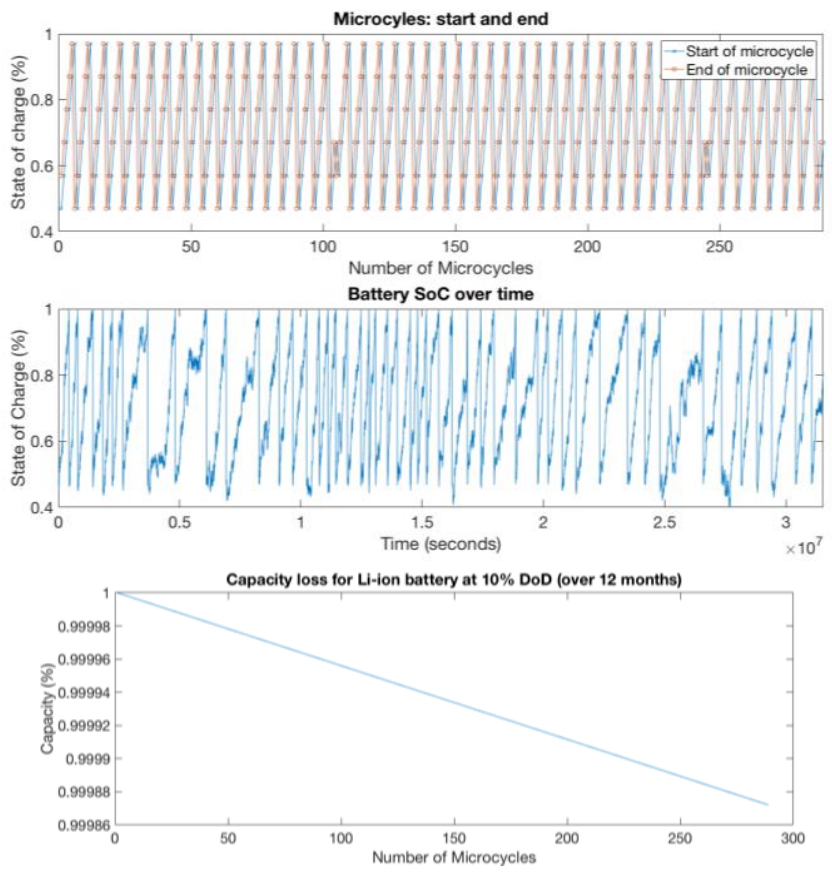

Fig 13 . Li-Ion battery with $10 \%$ DoD micro-cycles over a year 


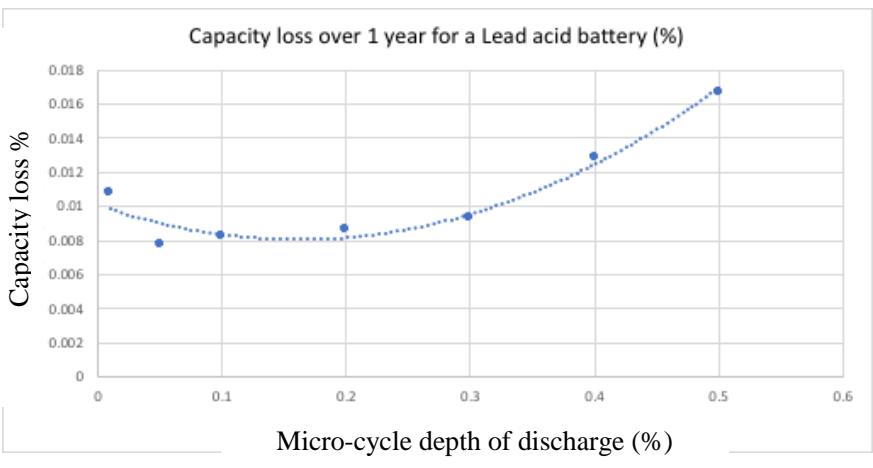

Fig 14. Micro-cycle depth vs calculated year loss of capacity for a lead acid battery

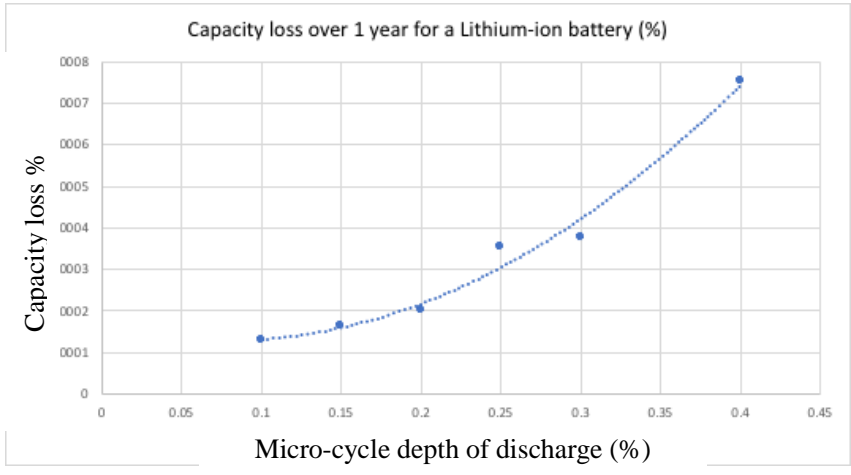

Fig 15. Micro-cycle depth vs calculated year loss of capacity for a Li-Ion battery

The impact of varying the definition of the micro-cycle depth is a variation in loss of capacity from $1 \%$ for a microcycle DOD of $1 \%$, through to $1.6 \%$ for a DOD micro-cycle of $5 \%$. This is less of an issue with a Li-Ion battery as shown in Fig 15, which shows a variation of only $0.05 \%$.

\section{CONCLUSIONS}

This paper has looked at different methods of determining the battery loss of life due to micro-cycles within a frequency response application. The main issues reported are that the micro-cycles are so small under normal operation that it is difficult to establish an accurate loss of life or capacity from traditional means of calculation. An alternative method using different fixed DOD cycles has been used to estimate the sort of variation that may be present. Results show that although degradation due to micro-cycling appears to be small, the variation in its value especially in batteries with low total life cycles may be significant. This stresses the need for more research in this area.

This is particularly important if hybrid systems are to be established which use a mixture of long life and reduced life batteries. These hybrid schemes offer the opportunity to reduce the cost of a battery system while providing a service. However, knowledge of their respective life spans is needed to properly size each part of the system and decide where in the operating regime each battery type is to operate.

\section{REFERENCES}

[1] National Grid, "Mandatory Frequency Response," [Online]. Available: http://www2.nationalgrid.com/uk/services/balancingservices/frequency-response/mandatory-frequency-response/. [Accessed 13 September 2016].

[2] J.C.M. Vieira, "Formulas for predicting the dynamic performance of ROCOF relays for embedded generation applications," IEE Proc.-Gener. Transm. Distrib, vol. 153, no. 4, p. 2, 2006.

[3] National Grid, "Enhanced Frequency Response," 26 August 2016. [Online]. Available: http://www2.nationalgrid.com/EnhancedFrequency-Response.aspx. [Accessed 14 September 2016].

[4] National Grid, "Future frequency response products", [Online], Avail;able at https://www.nationalgrid.com/uk/electricity/balancingservices/frequency-response-services/future-frequency-responseproducts [Accessed 21/05/18]

[5] Gundogdu, B., Nejad, S., Gladwin, D.T. "A Battery Energy Management Strategy for UK Enhanced Frequency Response" IEEE: 26th International Symposium on Industrial Electronics. 19-21 Jun 2017,Edinburgh, UK. IEEE , pp. 26-31.

[6] A. Cooke, D. Strickland and K. Forkasiewicz, "Energy storage for enhanced frequency response services," 2017 52nd International Universities Power Engineering Conference (UPEC), Heraklion, 2017, pp. 1-6.

[7] Battery and Energy technologies [Online] Available from http://www.mpoweruk.com/life.htm. Accessed 21/05/17

[8] PowerTech systems [Online] Available from https://www.powertechsystems.eu/home/tech-corner/lithium-ironphosphate-lifepo4/ Accessed 21/05/17

[9] [9] IEC 61982:2012 "Secondary batteries (except lithium) for the propulsion of electric road vehicles - Performance and endurance tests"

[10] V. Sauvant-Moynot, J. Bernard, R. Mingant, A. Delaille, F. Mattera, S. Mailley,J.-L. Hognon and F. Huet, "ALIDISSI, a Research Program to Evaluate Electrochemical Impedance Spectroscopy as a $\mathrm{SoC}$ and $\mathrm{SoH}$ Diagnosis Tool for Li-ion Batteries", Oil \& Gas Science and Technology - Rev. IFP, Vol. 65 (2010), No. 1, pp. 79-89

[11] Weiping Liu; Charles Delacourt; Christophe Forgez; Serge Pelissier, "Study of graphite/NCA Li-ion cell degradation during accelerated aging tests - Data analysis of the SIMSTOCK project", 2011 IEEE Vehicle Power and Propulsion Conference,Year: 2011

[12] A.J Ruddell,.G Dutton, H Wenzl, C Ropeter, D.USauer, JMerten, COrfanogiannis, J.WTwidell, PVezin, "Analysis of battery current microcycles in autonomous renewable energy systems", Journal of Power Sources, Volume 112, Issue 2, 14 November 2002, Pages 531-546

[13] Dirk Magnor, Jochen Bernhard Gerschler, Madeleine Ecker, Peter Merk, Dirk Uwe Sauer, "Concept of a battery aging model for Lithium-Ion batteries considering the lifetime depndency ont eh operation strategy", 24th European Photovoltaic Solar Energy ConferenceAt: Hamburg, Germany. September 2009

[14] Bolun Xu, , Jinye Zhao, , Tongxin Zheng,, Eugene Litvinov, Daniel S. Kirschen, "Factoring the Cycle Aging Cost of Batteries Participating in Electricity Markets", IEEE Transactions on Power Systems,2018, vol33, issue 2,pp 2248-2259 